\title{
Ectopic hematopoiesis in the human iris
}

\author{
J. Reimer Wolter, Bertram Schnitzer \& Arthur Wieringa \\ Departments of Ophthalmology and Pathology of the University of Michigan Medical Center, Ann Arbor, \\ Michigan, USA
}

Accepted 29 October 1990

Key words: iris, extramedullary hematopoiesis, precursor cells, erythroblastosis

\section{Summary}

Hematopoietic islands of erythroblastic precursor cells with mitotic activity were found pathologically in the distorted iris of a 35-year-old diabetic male. The eye had been enucleated for absolute hemorrhagic glaucoma occurring secondary to central vein occlusion and complicating previous vitrectomy, repair of retinal detachment and extracapsular cataract extraction.

\section{Introduction}

Hematopoiesis in adults is confined to the bone marrow and may be seen in extramedullary sites only in certain chronic myeloproliferative disorders. In the fetus and in premature infants, in contrast, ectopic hematopoiesis is usually present, predominantly in spleen and liver. Ectopic hematopoiesis, however, may also rarely be reactive, usually secondary to different forms of chronic anemia. Ectopic hematopoiesis, rarely, may be tumor-like in its appearance [3], and, finally, it may show a neoplastic potential (erythroblastoma).

It is the purpose of the present paper to demonstrate the pathology of tumor-like hematopoiesis in the iris stroma of the blind eye of an adult diabetic occurring in association with occlusion of the central retinal vein after lensectomy, repair of retinal detachment and use of a laser.

\section{Case report}

This 35 year-old white male with a 25 -year history of insulin-dependent diabetes was first seen in the
Ophthalmology Department of the University of Mich. on 11.9.1988, with vision of 20/400 in his left eye. A pinhole improved this to 20/100. The history revealed that he had not been born prematurely. As a college student the patient suffered a brain abscess. In October 1988, he developed progressive vision loss in his left eye. Examination on the Retina Service of this Ophthalmology Department revealed diabetic retinopathy with preretinal fibrosis and neovascularization with tractional retinal detachment involving the fovea. On 11.21.1988 a left closed vitrectomy with membrane peeling, lensectomy, application of endolaser, and air-fluid exchange were done with successful re-attachment of the retina. The intraocular pressure remained in the normal range throughout the operative and postoperative period. However, vision in the left eye was only light perception, when the patient was discharged from the hospital on 11.24.1988. Following this discharge the patient lost all vision in the left eye and the eye became very painful. The eye was enucleated on 1.31.1989. 


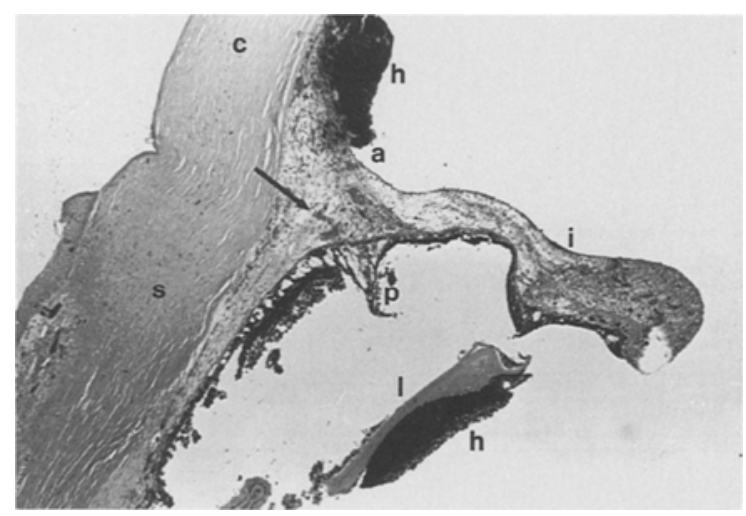

Fig. 1. Limbal cornea (c), closed angle (a) with hemorrhage (h), distorted iris (i) with cystic changes in the pigment epithelium (p), portion of peripheral lens capsule (l) with blood (h) on it, and anterior sclera (s). Arrow points to zone with ectopic hematopoietic cells. - Paraffin section, $\mathrm{H}$ and E stain, $\times 30$.

\section{Pathological findings}

The eye measured $23 \times 25 \times 26 \mathrm{~mm}$ and appeared approximately normal externally. It was opened in a horizontal plane. The anterior chamber was shallow and contained blood. The iris was distorted and dilated. The lens was absent. Brownish fluid ran out of the vitreous space. Retina and choroid were in place.

Microscopic study of paraffin sections showed

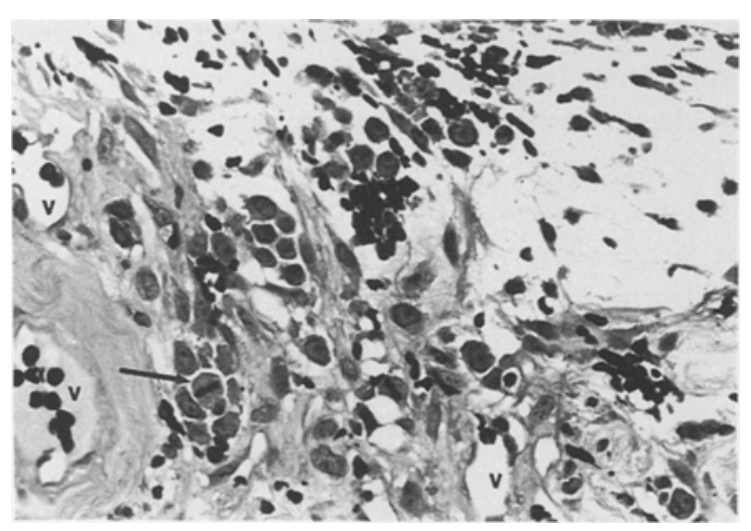

Fig. 3. Area of iris stroma with precursor cells in diffuse distribution with mitosis (arrow). Small iris vessels (v), cystoid changes and diffuse mononuclear infiltration are also seen. - Paraffin section, $H$ and $E$ stain, $\times 400$.

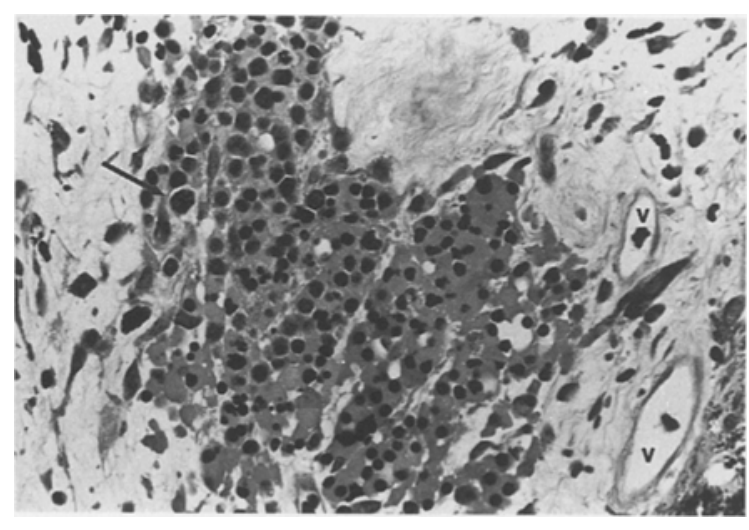

Fig. 2. Accumulation of erythroid precursor cells with mitosis in iris stroma (arrow). v: small iris vessels. - Paraffin section, $\mathrm{H}$ and $\mathrm{E}$ stain, $\times 400$.

extensive loss of corneal epithelium and associated corneal edema. Descemet's membrane was preserved. There was diffuse loss of corneal endothelial cells, but the endothelial layer was preserved. The angle was closed and exhibited neovascularization with scarring and associated anterior chamber hemorrhage (Fig. 1). The iris was distorted and showed neovascularization all over its anterior surface (Fig. 1). The pigment epithelium revealed degeneration and advanced cystoid changes (Fig. 1). Remnants of lens capsule were seen attached to the back of the iris (Fig. 1). Hemorrhages next to lens capsule remnants were explained by the recent surgery (Fig. 1). The iris stroma showed diffuse scarring and mononuclear cell infiltration. In its temporal portions the iris stroma contained accumulations of erythroid precursor cells in various stages of differentiation (Fig. 2). Between clusters of these cells, diffuse infiltration with erythroid precursor cells was seen (Fig. 3). Mitosis found in these cells was evidence of active proliferation (Fig. 3). Additional infiltration with macrophages and other mononuclear inflammatory cells was also seen in the iris. Some of the hematopoietic clusters were directly associated with branches of newly-formed, thin-walled iris blood vessels (Figs 2, 3).

The peripheral portions of the lens capsule were in contact with zonular fibers and directly adjacent to fresh hemorrhage in the anterior vitreous space 
(Fig. 1). The ciliary body showed diffuse mononuclear infiltration. The retina was in place and exhibited diffuse atrophy of its inner layers, hemorrhages, and scarring suspected to be a result of laser photocoagulation. The pigment epithelium was partly atrophic. The optic nerve exhibited recent occlusion of the central retinal vein. The choroid was involved with diffuse mononuclear infiltration and areas of atrophy. A diagnosis was made of absolute hemorrhagic glaucoma following central vein occlusion in an eye with hematopoiesis of the iris.

\section{Discussion}

Blood-forming cells in the human iris stroma have never before been described. However, ectopic hematopoiesis has been seen in the choroid and in remnants of the hyaloid system of the vitreous in premature and full-term newborn infants $[3,1]$. In these babies the hematopoiesis sometimes had tumor-like proportions [3]. Only in one baby has a relationship of the choroidal hematopoiesis to a general pathologic cause (erythroblastosis fetalis) been uncovered [3]. It was concluded that the choroid may be one of the physiologic sites for hematopoiesis during fetal life [3]. Ectopic (extramedullary) hematopoiesis in infants may also occur in the connective tissues of the orbit - especially around ciliary vessels [2] or in heterotopic bone marrow of the ocular region [3]. Secondary ectopic hematopoiesis in the eye region, may also occur in association with persistent anemia [3]. Extramedullary hematopoiesis of a non-tumorous type, finally, may also occur in the region of osseous metaplasia of the retinal pigment epithelium in advanced stages of phthisis [1].

In the present case the ectopic hematopoiesis in the iris stroma of an adult diabetic was not associated with anemia or any other significant primary or secondary disturbances of blood formation. It has already been pointed out that it may be difficult to recognize single hematopoietic precursor cells in the uvea among the elements of chronic inflammation [3]. However, the clusters of ectopic hematopoiesis in the iris of the present case were very distinct and would not have been mistaken for chronic inflammation by any pathologist.

\section{Acknowledgement}

Supported by The Research To Prevent Blindness, Inc., New York, N.Y.

\section{References}

1. Green WR. In Spencer, Ophthalmic Pathology, WB Saunders, Vol III pp 1424-1425, 1986.

2. Gilmour JR. Normal hematopoiesis in intrauterine and neonatal life. J Path \& Bact 1941; 52: 25-32.

3. Reese AB, Blodi FC. Hematopoiesis in and around the eye. Amer J Ophthalmol 1954: 38: 214-21.

Address for offprints:

J.R. Wolter,

Departments of Ophthalmology and Pathology,

University Medical Center,

Ann Arbor,

MI 48109-0602 1301 Catherine, USA 